Article

\title{
The Quasi Steady State Cosmology in a Radiation Dominated Phase
}

\author{
Raj Bali* \\ Department of Mathematics, University of Rajasthan, Jaipur-302004, India \\ ${ }^{*}$ Corresponding Author: Email: rbalijpr@gmail.com
}

Abstract: Analytical solutions for radiation dominated phase of Quasi Steady State Cosmology in Friedmann-Robertson-Walkar models are obtained. We find that matter density is positive in all the cases $(k=0,-1,1)$. The nature of Hubble parameter $(H)$ in $[0,2 \pi]$ is discussed. The deceleration parameter (q) is marginally less than zero indicating accelerating universe. The scale factor (S) is graphically shown with time. The model represents oscillating universe between the above mentioned limits. Because of bounce in QSSC, the maximum density phase is still matter dominated. The models represent singularity free model. We also find that the models have event horizon i.e. no observer beyond the proper distance $\mathrm{rH}_{\mathrm{H}}$ can communicate each other in FRW mdels for radiation dominated phase in the frame work of QSSC. The FRW models are special classes of Bianchi type I, V, IX space-times with zero, negative and positive curvatures respectively. Initially i.e. at $\tau=0$, the model represents steady model. We have tried to show how a good fit can be obtained to the observations in the framework of QSSC during radiation dominated phase.

Keywords: Quasi Steady State Cosmology; Radiation Phase

\section{Introduction}

In the early stages of the universe, the radiation was the dominant influence on the expansion of the universe because most of the energy was in the form of radiation. Thus examination of the radiation dominated expansion is essential for understanding the early universe. The main motivation to introduce QSSC is to have a universe without a singularity in which matter is created in an explosive way but physically understood (Narlikar, [1]). Any 
alternative cosmology must be more successful in explaining observations. In this cosmology the cosmological constant $(\lambda)$ is taken negative because we always get an oscillating universe whatever the curvature parameter. To understand the radiation dominated phase of universe, and QSSC, it is interesting to know about the Microwave Background Radiation (CMBR) and abundances of light nuclei in early universe. Between 1964 and 1965, the steady state model received two near-fatal blows. Both came from considerations of issues of the early universe, (i) the discovery of microwave background (ii) the abundances of light nuclei. We discuss these briefly in order.

The cosmic microwave background is considered to be left over radiation from the big bang or the time when the universe began (Space.com). As mentioned by Narlikar [1], the case of radiation dominated universe differs from the matter dominated one where the pressure $\mathrm{p}$ of radiation makes a significant contribution to gravitational attraction and in this case, we have $\rho=$ $3 p$ (the speed of light is considered as $c=1$ in gravitational unit). However, the turning point for cosmology came with the discovery of the microwave background radiation (Penzias and Wilson [2]). It confirmed the early universe scenario and taken together with the extended validity of Hubble's law. Also according to NASA observations, it is uniform temperature with small fluctuations visible with precise telescopes. Studying these fluctuations, cosmologists can learn about the origin and large scale structure of universe and can know how the early universe was formed.

The Gamow's ideas [3] on primordial nucleosynthesis had turned out to work for light nuclei only, producing mainly helium and small quantities of deuterium in stars and a few other light nuclei. For the majority of elements, stars provided the right setting for successive nucleosynthesis. This had led most astronomers to believe that stellar nucleosynthesis was the key process for all elements, since even helium was seen to be produced in stars. Apart from helium, the discovery of deuterium made the primordial version more credible since there was no 
known process for making even tiny quantities of deuterium in stars. So the steady state theory, which had no early hot era to make such light nuclei, faced difficulties in explaining their observed abundances. The case for the hot big bang become even stronger due to the discovery of microwave background. The steady state model had no natural process to maintain a radiation background at $\sim 3 \mathrm{~K}$ and it never quite recovered from these two blows. Nevertheless, the model made a comeback in a modified form in the 1990s.

In 1993, Hoyle, Burbidge and Narlikar introduced a new cosmology known as Quasi Steady State Cosmology (QSSC) which drew considerable interest on the earlier steady state cosmology. It also allows the possibility of evolution of universe on shorter time scale and arises from the consideration of Hoyle-Narlikar theory [4]. Hoyle et al. [4,5] and Burbidge et al. [6] reviewed the progress of QSSC with the intention to bring a viable alternative to the standard hot big bang cosmology (HBBC). The HBBC has two versions: (i) the version in which the universe expands from a singularity in a radiation dominated phase which changes over to matter dominated one (ii) Post 1981 version in which there is a brief description of inflation very early in a radiation dominated phase. The later version was also called inflationary Big Bang Cosmology (IBBC) which was proposed to get rid of some of the conceptual defects of HBBC. While it was partially successful but it had problems of its own as explained by Narlikar and Padmanabhan [7]. Using the field equations of QSSC, Sachs et al. [8] obtained the analytical solutions which relate with the observations. Some developments of quasi steady cosmology has been summarized by Narlikar [9].

Riess et al. [10] and Perlmutter et al. [11] in their investigations used type Ia supernovae and have shown that the universe is accelerating. This then is a result which was predicted by QSSC and it has been shown by Banerjee et al. [12] that a good fit can be made to the observed data using QSSC. Burbidge [13] has pointed out that the decelerating parameter (q) is negative in quasi steady state cosmology. Thus this observational evidence confirms a real prediction in 
favour of QSSC. Narlikar et al. [14] have mentioned that cosmological observations support the accelerating models of the universe as driven by a cosmological constant or dark energy. It is also well established that the universe is expanding at an accelerating rate (Hawking and Mlodinow [15],[16], Krauss [17], Bali and Saraf [18,19] Bali and Singh [20].

\section{The Metric and Field Equations}

We consider homogeneous and isotropic space-time of Robertson-Walker in the form given by

$$
\mathrm{ds}^{2}=\mathrm{c}^{2} \mathrm{dt}^{2}-\mathrm{S}^{2}(\mathrm{t})\left[\frac{\mathrm{dr} \mathrm{r}^{2}}{1-\mathrm{kr}^{2}}+\mathrm{r}^{2} \mathrm{~d} \theta^{2}+\mathrm{r}^{2} \sin ^{2} \theta \mathrm{d} \phi^{2}\right]
$$

where $S(t)$ is scale factor and $k=0,-1,1$.

The modified Einstein's field equations for QSSC is considered in the orm

$$
\mathrm{R}_{\mathrm{ik}}-\frac{1}{2} \mathrm{Rg}_{\mathrm{ik}}+\lambda \mathrm{g}_{\mathrm{ik}}=-8 \pi \mathrm{G}\left[\mathrm{T}_{\mathrm{ik}}-\mathrm{f}\left\{\mathrm{C}_{\mathrm{i}} \mathrm{C}_{\mathrm{k}}-\frac{1}{4} \mathrm{~g}_{\mathrm{ik}} \mathrm{C}^{\ell} \mathrm{C}_{\ell}\right]\right.
$$

where $\lambda=-3 \Lambda \mathrm{m}_{0}^{2}$, is cosmological constant, $\mathrm{f}>0$ the coupling constant between matter and creation field in conventional unit. the constant $\Lambda$ is the square of the reciprocal of the primary Planck particles and $\Lambda>0$.

For the metric (1), the field equation (2) leads to

$$
\begin{aligned}
& 2 \frac{\ddot{\mathrm{S}}}{\mathrm{S}}+\frac{\dot{\mathrm{S}}^{2}}{\mathrm{~S}^{2}}+\frac{\mathrm{k}}{\mathrm{S}^{2}}=3 \lambda-8 \pi \mathrm{Gp}+2 \pi \mathrm{Gf} \dot{\mathrm{C}}^{2} \\
& 3 \frac{\dot{\mathrm{S}}^{2}}{\mathrm{~S}^{2}}+\frac{3 \mathrm{k}}{\mathrm{S}^{2}}=3 \lambda+8 \pi \mathrm{G} \rho-6 \pi \mathrm{Gf} \dot{\mathrm{C}}^{2}
\end{aligned}
$$

\section{Solution of Field Equations}

We assume that the universe is dominated by radiation phase. Thus, we have $\rho=3 p$ which leads to 


$$
\frac{6 \ddot{\mathrm{S}}}{\mathrm{S}}+\frac{6 \dot{\mathrm{S}}^{2}}{\mathrm{~S}^{2}}+\frac{6 \mathrm{k}}{\mathrm{S}^{2}}-12 \lambda=0
$$

which leads to

$$
2 \ddot{\mathrm{S}}+\frac{2 \dot{\mathrm{S}}^{2}}{\mathrm{~S}}=-\frac{2 \mathrm{k}}{\mathrm{S}}+4 \lambda \mathrm{S}
$$

The equation (6) leads to

$$
\begin{aligned}
& \frac{\mathrm{d}}{\mathrm{dS}}\left(\mathrm{F}^{2}\right)+\frac{2}{\mathrm{~S}} \mathrm{~F}^{2}=-\frac{2 \mathrm{k}}{\mathrm{S}}+4 \lambda \mathrm{S} \\
& \quad \text { where } \dot{\mathrm{S}}=\mathrm{F}(\mathrm{S}), \ddot{\mathrm{S}}=\mathrm{FF}^{\prime}, \mathrm{F}^{\prime}=\frac{\mathrm{dF}}{\mathrm{dS}}
\end{aligned}
$$

Solution of (7) is given by

$$
\begin{aligned}
& \mathrm{F}^{2} \cdot \mathrm{S}^{2}=-2 \mathrm{k} \int \mathrm{SdS}+4 \lambda \int \mathrm{S}^{3} \mathrm{dS} \\
& =-\mathrm{k} \mathrm{S}^{2}+\lambda \mathrm{S}^{4}+\alpha
\end{aligned}
$$

where $\alpha>0$, is constant of integration. Equation (8) leads to

$$
\mathrm{F}=\left(\frac{\mathrm{d} S}{\mathrm{dt}}\right)= \pm\left(\frac{\alpha+\lambda \mathrm{S}^{4}-\mathrm{kS} \mathrm{S}^{2}}{\mathrm{~S}^{2}}\right)^{1 / 2}
$$

Thus, we have

which leads to

$$
\frac{-2 \mathrm{SdS}}{\sqrt{\alpha-3 \Lambda \mathrm{m}_{0}^{2}} \mathrm{~S}^{4}-\mathrm{kS} \mathrm{S}^{2}}=2 \mathrm{dt}
$$

$$
\frac{-2 S d S}{\sqrt{\alpha-3 \Lambda m_{0}^{2}\left(S^{2}+\frac{k}{6 \Lambda m_{0}^{2}}\right)^{2}+\frac{k^{2}}{36 \Lambda^{2} m_{0}^{4}}}}=2 d t
$$

Thus, we have 


$$
\frac{-2 \mathrm{SdS}}{\sqrt{\left(\frac{\alpha}{3 \Lambda \mathrm{m}_{0}^{2}}+\frac{\mathrm{k}^{2}}{36 \Lambda^{2} \mathrm{~m}_{0}^{4}}\right)}-\left(\mathrm{S}^{2}+\frac{\mathrm{k}}{6 \Lambda \mathrm{m}_{0}^{2}}\right)^{2}}=2 \sqrt{3 \Lambda \mathrm{m}_{0}^{2}} \mathrm{dt}
$$

Using

$$
\mathrm{S}^{2}+\frac{\mathrm{k}}{6 \Lambda \mathrm{m}_{0}^{2}}=\xi
$$

Thus, we have

$$
2 \mathrm{SdS}=\mathrm{d} \xi
$$

Now equation (10) leads to

$$
\frac{-\mathrm{d} \xi}{\sqrt{\mathrm{a}^{2}-\xi^{2}}}=2 \sqrt{3 \Lambda \mathrm{m}_{0}^{2}} \mathrm{dt}
$$

where

$$
\mathrm{a}^{2}=\frac{\alpha}{3 \Lambda \mathrm{m}_{0}^{2}}+\frac{\mathrm{k}^{2}}{36 \Lambda^{2} \mathrm{~m}_{0}^{4}}
$$

Equation (11) leads to

$$
\cos ^{-1} \frac{\xi}{\mathrm{a}}=2 \sqrt{3 \Lambda \mathrm{m}_{0}^{2}}\left(\mathrm{t}+\mathrm{t}_{0}\right)
$$

where $t_{0}$ is constant.

Thus, we have

$$
\xi=a \cos \left(\sqrt{3 \Lambda m_{0}^{2}} \tau\right)
$$

where $t+t_{0}=\tau$. Therefore, we have

$$
\mathrm{S}^{2}+\frac{\mathrm{k}}{6 \Lambda \mathrm{m}_{0}^{2}}=\mathrm{a} \cos \left(\sqrt{3 \Lambda \mathrm{m}_{0}^{2}} \tau\right)
$$

i.e. 


$$
\mathrm{S}^{2}=-\frac{\mathrm{k}}{6 \Lambda \mathrm{m}_{0}^{2}}+\sqrt{\frac{\alpha}{3 \Lambda \mathrm{m}_{0}^{2}}+\frac{\mathrm{k}^{2}}{36 \Lambda^{2} \mathrm{~m}_{0}^{4}}} \cos \left(\mathrm{m}_{0} \sqrt{3 \Lambda} \tau\right)
$$

where $3 \Lambda \mathrm{m}_{0}^{2} \simeq 10^{-56} \mathrm{~cm}^{-2}$ in conventional unit.

Let $\alpha=1 / 3 \Lambda \mathrm{m}_{0}^{2}$, then

$$
\begin{gathered}
\mathrm{k}=0, \mathrm{~S}^{2}=1 \times 10^{56} \operatorname{Cos}\left(10^{-28} \tau\right] \\
\mathrm{k}=-1, \mathrm{~S}^{2}=5 \times 10^{55}+1.1 \times 10^{56} \operatorname{Cos}\left[10^{-28} \tau\right] \\
\mathrm{k}=1, \mathrm{~S}^{2}=-5 \times 10^{55}+1.1 \times 10^{56} \operatorname{Cos}\left[10^{-28} \tau\right]
\end{gathered}
$$

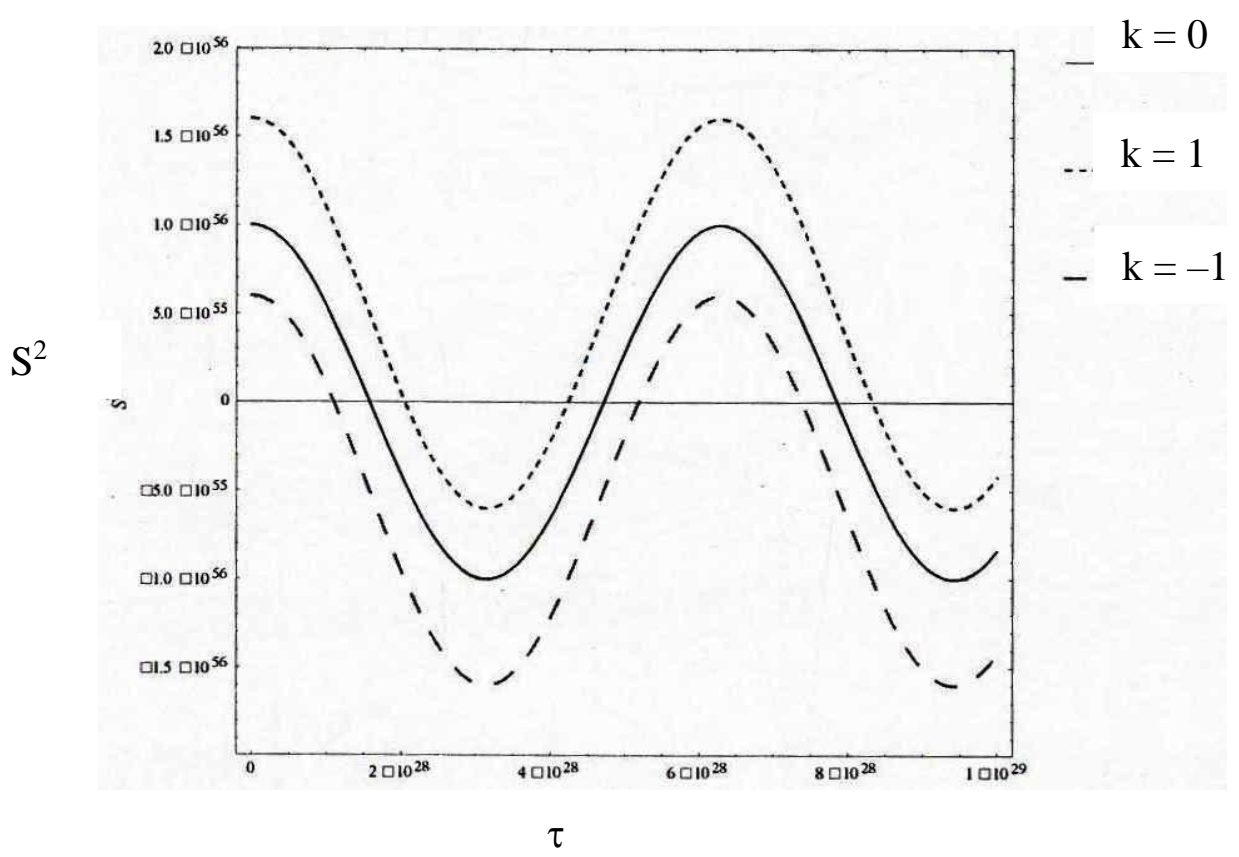

Case (i): If $\mathrm{k}=0$ then equation (14) leads to

$$
\begin{aligned}
& \mathrm{S}^{2}=\frac{1}{3 \Lambda \mathrm{m}_{0}^{2}} \cos \left(\sqrt{3 \Lambda \mathrm{m}_{0}^{2}} \tau\right) \\
& =10^{56} \cos \left(10^{-28} \tau\right)
\end{aligned}
$$

Case (ii): If $\mathrm{k}=-1$ then equation (14) leads to

$$
\mathrm{S}^{2}=\frac{1}{2\left(3 \Lambda \mathrm{m}_{0}^{2}\right)}+\sqrt{\frac{1}{\left(3 \Lambda \mathrm{m}_{0}^{2}\right)^{2}}+\frac{1}{4\left(3 \Lambda \mathrm{m}_{0}^{2}\right)^{2}}} \cos \left(\sqrt{3 \Lambda \mathrm{m}_{0}^{2}} \tau\right)
$$




$$
\begin{gathered}
=0.5 \times 10^{56}+\sqrt{10^{112}+\frac{1}{4} 10^{112}} \cos \left(\sqrt{3 \Lambda \mathrm{m}_{0}^{2}} \tau\right) \\
=0.5 \times 10^{56}+10^{56} \sqrt{\frac{5}{4}} \cos \left(\sqrt{3 \Lambda \mathrm{m}_{0}^{2}} \tau\right) \\
=0.5 \times 10^{56}+(1.1) \times 10^{56} \cos \left(10^{-28} \tau\right) \\
\simeq 2 \cos ^{2}\left(\frac{\left(10^{-28} \tau\right)}{2}\right)
\end{gathered}
$$

Case (iii): If $k=1$, then equation (14) leads to

$$
\begin{aligned}
& \mathrm{S}^{2}=-\frac{1}{2\left(3 \Lambda \mathrm{m}_{0}^{2}\right)}+\sqrt{\frac{1}{\left(3 \Lambda \mathrm{m}_{0}^{2}\right)^{2}}+\frac{1}{4\left(3 \Lambda \mathrm{m}_{0}^{2}\right)}} \cos \left(10^{-28} \tau\right) \\
& =-0.5 \times 10^{56}+\sqrt{10^{112}+\frac{1}{4} \times 10^{112} \cos \left(10^{-28} \tau\right)} \\
& =-0.5 \times 10^{56}+10^{56} \sqrt{\frac{5}{4}} \cos \left(10^{-28} \tau\right) \\
& =-0.5 \times 10^{56}+(1.1) \times 10^{56} \cos \left(10^{-28} \tau\right) \\
& \simeq 2 \sin ^{2}\left(\frac{\left(10^{-28} \tau\right)}{2}\right)
\end{aligned}
$$

For $\mathrm{k}=\mathbf{0}$

Hubble parameter $=\frac{\dot{S}}{S}=\tan \left(10^{-28} \tau\right) \times \frac{10^{-28}}{2}$

$$
=5 \times 10^{-29} \tan \left(10^{-28} \tau\right)
$$

Deceleration parameter $q=-\frac{\ddot{S} / S}{\dot{S}^{2} / S^{2}}$

$$
\frac{\dot{\mathrm{S}}}{\mathrm{S}}=5 \times 10^{-29} \tan \left(10^{-28} \tau\right)
$$




$$
\begin{gathered}
\frac{\ddot{S}}{\mathrm{~S}}-\frac{\dot{\mathrm{S}}^{2}}{\mathrm{~S}^{2}}=5 \times 10^{-29} \sec ^{2}\left(10^{-28} \tau\right) 10^{-28} \\
\therefore \frac{\ddot{\mathrm{S}}}{\mathrm{S}}=5 \times 10^{-57} \sec ^{2}\left(10^{-28} \tau\right)+25 \times 10^{-58} \tan ^{2}\left(10^{-28} \tau\right) \\
=50 \times 10^{-58} \sec ^{2}\left(10^{-28} \tau\right)+25 \times 10^{-58}\left(\sec ^{2} 10^{-28} \tau-1\right) \\
=75 \times 10^{-58} \sec ^{2}\left(10^{-28} \tau\right)-25 \times 10^{-58}
\end{gathered}
$$

Thus, equation (19) leads to

$$
\begin{aligned}
& \mathrm{q}=-\frac{\left[75 \times 10^{-58} \sec ^{2}\left(10^{-28} \tau\right)-25 \times 10^{-58}\right.}{25 \times 10^{-58} \tan ^{2}\left(10^{-28} \tau\right)} \\
& <0
\end{aligned}
$$

\section{Event Horizon}

The coordinate distance to the horizon $\mathrm{rH}(\tau)$ is the maximum distance a null ray could have travelled at time $\tau$ starting from the infinite past. Thus, we have

$$
\mathrm{r}_{\mathrm{H}}=\int_{-\infty}^{\infty} \frac{\mathrm{d} \tau}{\mathrm{S}(\tau)}
$$

We could extend the proper time $\tau$ to $(-\infty)$ in the past because of the non-singular nature of the space-time. Now, we have

For $\mathrm{k}=1$

$$
\begin{gathered}
r_{H}(\tau)=\int_{-\infty}^{\infty} \frac{d \tau}{S(\tau)} \\
\simeq \int_{0}^{\infty} \frac{d \tau}{\sqrt{2} \cos \left(\frac{10^{-28} \tau}{2}\right)} \\
\simeq \frac{1}{\sqrt{2}} \sec \left(\frac{\left(10^{-28} \tau\right)}{2}\right) d \tau
\end{gathered}
$$




$$
\simeq\left[\log \left[\sec \left(\frac{10^{-28} \tau}{2}\right)\right]+\tan \left(\frac{10^{-28} \tau}{2}\right)\right]_{0}^{\infty}
$$

For $\mathrm{k}=-1$

$$
\begin{gathered}
\mathrm{r}_{\mathrm{H}}(\tau)=\int_{-\infty}^{\infty} \frac{\mathrm{d} \tau}{\mathrm{S}(\tau)} \\
\simeq \int_{0}^{\infty} \operatorname{cosec}\left(\frac{10^{-28} \tau}{2}\right) \mathrm{dt} \\
\simeq \frac{1}{\sqrt{2}}\left[\log \tan \left(\frac{10^{-28} \tau}{4}\right)\right]_{0}^{\infty}=\infty
\end{gathered}
$$

For $\mathrm{k}=0$

$$
r_{H}(\tau)=\int_{-\infty}^{\infty} \frac{d \tau}{S^{2}(\tau)}=\infty
$$

as for case $\mathrm{k}=1$

In all the cases, we find that the models have event horizon i.e. no observer beyond the proper distance rH can communicate each other in FRW models in QSSC.

Following Hoyle and Narlikar (1964) in creation field cosmology, we have assumed $\dot{\mathrm{C}}=1$. Thus the matter density for the case $\mathrm{k}=0$, is given by $8 \pi \mathrm{G} \rho=3 / 410^{-56} \tan ^{2}\left(10^{-28} \tau\right)+6 \pi \mathrm{Gf}+3 \times 10^{-56}$. Similarly for $\mathrm{k}=1,-1$, the density can be calculated.

\section{Discussion and Conclusion}

The scale factor in all the cases $(k=0,1,-1)$ is shown graphically which gives the nature of scale factor in QSSC. The deceleration parameter (q) is marginally $<0$ which shows that the model represents accelerating phase of universe. Thus the observational evidence as investigated by Riess et al. (1998) and Perlmutter et al. (1999) using type Ia supernovae confirms a real 
prediction in favor of QSSC. The Hubble parameter $(\mathrm{H})$ for $\mathrm{k}=0$, is initially zero at $\tau=0$, maximum at $\quad \tau=\frac{\pi}{10^{-28}}$, zero at $\quad \tau=\frac{\pi}{10^{-28}}$, mximum at $\tau=\frac{3 \pi}{2 \times 10^{-28}}$ and zero at $\quad \tau=\frac{2 \pi}{10^{-28}}$. Similar case for $\mathrm{k}=1,-1$ may be discussed. Thus the models oscillate between these limits. Because of bounce in QSSC, the maximum density phase is still matter dominated. The bouncing universe is consistent with acceleration. Development of QSSC theory indicates that newly created particles have a mass $\left(\frac{3 h c}{4 \pi G}\right)^{1 / 2}$. Such Planck particles are unstable over a time scale $10^{-43} \mathrm{~s}$ (Hoyle et al. 1993). Presently, we are not in the radiative phase but it is interesting to study the Universe in radiative phase because in early stage of the Universe, most of the energy was in the form of radiation. Thus, examination of radiation dominated expansion is essential for understanding the early universe within QSSC in which matter is created in an explosive way (Narlikar 2012). The bouncing nature is involved in QSSC theory. The models represent singularity free space-times. The matter density ( $\rho$ ) is positive in all cases. The present model initially i.e. $\tau=0$ represents steady state model and at $\tau=0, \quad \frac{\pi}{10^{-28}}$ in $[0, \pi]$. The model has event horizon i.e. no observer beyond the proper distance $\mathrm{rH}_{\mathrm{H}}$ can communicate each other in FRW models for radiation dominated phase in QSSC.

\section{References}

1. J.V. Narlikar, "Introduction to Cosmology", Cambridge University Press, p.133, 2012.

2. A.A. Penzias and R.W. Wilson, "A measurement of Excess Antenna Temperature at 4080 Mc/s", Astrophys. J. Vol.142, pp.419-421, 1965.

3. B. Gamow, “Expanding Universe and the origin of elements" Phys. Rev. Vol. 70, pp. 572-573, 1946.

4. F. Hoyle and J. V. Narlikar, “A new theory of gravitation”, Proc. Roy. Soc. A, Vol.282, 1964, pp.191$207,1964$.

5. F. Hoyle, G. Burbidge and J.V. Narlkar, “A Quasi-Steady State Cosmological model with creation of matter", Astrophys. J., Vol.410, pp.437-457, 1993. 
6. G. Burbidge, F. Hoyle and J.V. Narlikar, "Further Astrophysical Quantities expected in a quasisteady state universe", Astronomy \& Astrophysics, Vol.289, 1994, pp.729-739, 1994

7. J.V. Narlikar and T. Padmanabhan, "Inflation for Astronomers", Annu. Rev. Astronomy, Astrophys., Vol.29, pp. 325-362, 1991.

8. R. Sachs, J.V. Narlikar and F. Hoyle, “The Quasi-Steady State Cosmology : Analytical solutions of field equations and their relationship to observations", Astronomy and Astrophys., Vol.313, pp.703$712,1996$.

9. J.V. Narlikar, "The Quasi-steady state cosmology : Some recent development" J. Astrophys. Astronomy, Vol.18, p.353,363, 1997.

10. A.G. Riess et al., "Observational evidence from Supernovae for an accelerating universe and a cosmological constant", Astron. J. Vol.116, 1998, pp.1009-1038, 1998.

11. S. Perlmutter et al., "Measurements of $\Omega$ and $\Lambda$ from 42 high redshift supernovae", Astrophys. J., Vol.517, pp.565-586, 1999.

12. S.K. Banerjee, J.V. Narlikar, N.C. Wickramsinghe, F. Hoyle and G. Burbidge, "Possible interpretations of the magnitude-redshift relation for supernovae of Type IA", The Astrophysical Journal, 119, pp.2583-2588, 2000.

13. G. Burbidge, “Quasi-steady state cosmology”, arxiv;astro-ph/0108051v1, 2001.

14. J.V. Narlikar, R.G. Vishwakarma and G. Burbidge, "Interpretation of the accelerating universe", The Astronomical Society of the Pacific, Vol.114, pp.1092-1096, 2002.

15. S.W. Hawking and L. Mlodinow, "A brief history of time”, Bantam Dell, Random House, Inc., New York, 2005.

16. S.W. Hawking and L. Mlodinow, “The Grand Design”, Bantam Books, Random House, Inc., New York, 2010

17. L.M. Krauss, “A universe from nothing”, Free Press, Simon and Schuster, New York, 2012.

18. R. Bali and S. Saraf, "C-field Cosmological model for barotropic fluid distribution with varying $\Lambda$ in FRW space-time", Int. J. Theor. Phys. Vol.52, pp.1645-1650, 2013. 
19. R. Bali and S. Saraf, "C-field cosmological model for barotropoic fluid distribution with bulk viscosity and decaying vacuum energy $(\Lambda)$ in FRW space-time", Canadian J. Phys. Vol.91, pp.1-5 (2015).

20. R. Bali and S. Singh, "Inflationary scenario in Bianchi Type V space-time for barotropic fluid with variable bulk viscosity and vacuum energy density", Gravitation and Cosmology, Vol.16, pp.394403, 2016. 